\title{
Adaptive control over ultrasonic cleaning of mining equipment
}

\author{
Volodymyr Morkun, and Olha Kravchenko ${ }^{1 *}$ \\ ${ }^{1}$ Kryvyi Rih National University, Department of Automation, Computer Sciences and Technology, \\ 11 Matusevycha St., 50027 Kryvyi Rih, Ukraine
}

\begin{abstract}
Ultrasonic cleaning is one of the most efficient types of cleaning mining equipment. In order to enhance its energy efficiency, it is required to improve control over ultrasonic cleaning through considering its spacious distribution. This control is based on assessing the condition of a cleaned object in set points to determine duration of the process according to the real contamination of a product. In order to form controlling impact, the authors suggest a new fuzzy controller, which combines features of a $3 \mathrm{D}$ fuzzy controller and an interval fuzzy controller of Type 2. This enables taking advantages of both extensions of fuzzy logic - an opportunity to process spacious data through fuzzy expert evaluation. The concept of a controller is based on the 3-D interval fuzzy set of Type 2 . The developed controller differs from the traditional one by reduced dimension and reduction of IT2 FS to IT1 FS. The rule base remains two-dimensional and is not dependent on the number of sensors. The elaborated methods enable the controller model based on 3-D IT2 FS for ultrasonic cleaning of mining equipment. Spacious distribution of the process and ambiguity of expert assessments are considered to determine the process course according to the data of ultrasonic sensors.
\end{abstract}

\section{Introduction}

Efficient cleaning is essential for improving durability of mining equipment and enables its current repairs. Various methods of equipment cleaning are applied to mining. Chemical cleaning requires employment of hazardous chemical solvents, while mechanical cleaning is noted for low efficiency in spite of great efforts applied. Unlike them, ultrasonic cleaning is one of the most efficient and promising types of cleaning as it enables satisfactory results without using harmful chemical solvents and hard physical labour. Yet, automation of ultrasonic cleaning remains quite primitive nowadays and most ultrasonic baths are able to control only time indices. For this reason, developing automation methods to control ultrasonic cleaning in a more efficient way is an urgent, yet complicated task. The complex character of this problem is conditioned by a great number of factors affecting ultrasonic cleaning including a contamination type, temperature of washing solution, its composition, ultrasound capacity and frequency, size and form of a tank, position of an ultrasonic transducer, etc.

\footnotetext{
*Corresponding author: kravchenko@knu.edu.ua
} 
Many researches have been aimed at improving efficiency of the basic component of ultrasonic cleaning and cavitation collapses depending on a variety of factors. Thus, [1] considers impact of oxygen concentration on the number of cavitation bubbles, while [2] investigates into dependency of cavitation on liquid temperature and degasing, and in [3] a new method of measuring cavitation activity enables monitoring its zoning. [4] also deploys a new approach to measuring cavitation depending on various factors, in particular, dependency of liquid temperature and gas contamination, frequency, intensity and time of insonation.

Thus, ultrasonic cleaning is difficult to predict as too many interrelated factors affect it. Besides, the process is characterized by spacious distribution caused by some physical characteristics of cavitation. Spacious distribution is also conditioned by the cleaned object itself that can have an arbitrary form and irregular contamination. Available systems of ultrasonic cleaning automation ignore this factor considering only the liquid condition to determine the process duration [5-6]. In this case, there are power excess demands as ultrasonic cleaning occurs in cleaned sections as well. In order to improve energyefficiency of ultrasonic cleaning, one should control the process through assessing the condition of a cleaned body. This can be done through analyzing ultrasonic reflections recorded by sensors in set points. Ultrasonic cleaning modelling can be complicated by absence of a mathematical equation describing cavitation processes and cleaning considering geometry of a cleaned body.

For this reason, modelling of ultrasonic cleaning is reduced to determining ultrasonic pressure in each spacious point at a certain time unit and assessing zones with abrupt pressure differences causing cavitation collapses [7 - 9]. This approach enables forecasting only possible available cavitation collapses and is unable to assess the course of the cleaning process as it ignores the influence of ultrasonic radiation on the product under cleaning.

Modelling by means of the k-wave software [10-12] has detected inability to determine factors affecting changes in sensor signals at the given moment - separation of a contaminated layer or a body's geometry. There are no clearly defined changes of a signal for bodies of various forms. Thus, in assessing the ultrasonic cleaning process, we consider the following assumptions:

1. There are no data on the form of a cleaned body and the rate of its contamination. This will not require initial expert evaluation.

2. The process can be aimed at cleaning only, i.e. any changes in a signal indicate separation of dirt. Therefore, it is reasonable to stop ultrasonic cleaning if there are no considerable changes on all the sensors during the set period.

3. The sensor closest to the separated dirt records the greatest change of the signal.

These assumptions determine the basis for assessing the course of ultrasonic cleaning through analyzing ultrasonic responses in the cleaning tank.

\section{Configuration of the 3-D IT2 FS controller}

Many physical processes including ultrasonic cleaning are referred to the systems with distributed parameters. In practice, the distributed character of these systems is ignored [13], as traditional control methods are applied to systems with concentrated parameters. Yet, modern industry requires higher standards of energy-efficiency and safety [14-16]. Traditional control methods for systems with concentrated parameters cannot meet these requirements. In recent decades, traditional control methods for systems with concentrated parameters [17 - 19] have been expanded. Yet, they require complicated mathematical models based on differential equations in partial derivatives, integral equations, integraldifferential equations or sets of various equations. More up-to-date methods [20 - 23] based 
on system training and applied expert experience are used to avoid the problem of too complicated and bulky mathematical models. Thus, in [24], control is based on noninvasive assessment of contamination of tubes by means of convolutional neural networks. Yet, considering a great variety of mining machines, training algorithms for cleaning require a great amount of calculation and time. 3-D fuzzy control is deprived of this drawback and envisages formation of control considering system distribution [25-26]. This is achieved by applying a 3-D fuzzy controller, which due to a fuzzy set with an additional spacious coordinate is able to process spacious data. Application of this controller solves the problem of spacious distribution of the ultrasonic cleaning system, yet we cannot obtain a clear conclusion on the contamination rate of mining machines. Duration of the process is determined by assessing changes of ultrasonic responses in the cleaning tank. Expert evaluation of this parameter is noted for its wide range of values. This problem is solved by applying the interval membership function that enables expert evaluation in the form of a range.

Thus, the 3-D fuzzy interval controller combines a 3-D fuzzy controller [25] and an interval fuzzy controller of Type 2 [27-29]. This enables combining the following advantages of both expansions of fuzzy logic - processing spacious data and considering spacious distribution of the system as well as ambiguity of expert evaluation of the membership function. The controller is based on a 3-D interval fuzzy set of Type 2 .

The 3-D interval fuzzy set of Type 2 (3-D IT2 FS) is determined as an expansion of the 3-D fuzzy set, the membership function of which is interval.
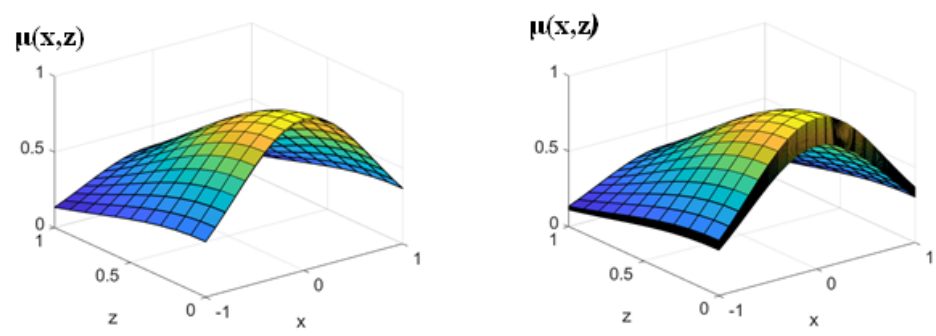

Fig. 1. Fuzzy sets a - a 3-D fuzzy set; b - a 3-D interval fuzzy set of Type 2.

In other words, we determine the 3-D interval fuzzy set of Type $2 \tilde{A}$ through the content variable $X$, the unidimensional space variable $Z$ and the fuzzy membership function $\mu(\mathrm{x}, \mathrm{z})$ (Fig. 1).

$$
\begin{aligned}
& \tilde{A}=\left\{(x, z), \mu_{\tilde{A}}(x, z) \mid \forall x \in X, z \in Z\right\}, \\
& \mu_{\tilde{A}}(x, z)=\int_{u} \frac{1}{u}, u \in J_{x} \subseteq U=[0,1],
\end{aligned},
$$

where $J_{x}$ is a primary membership, $\mathrm{U}$ is the domain of primary memberships, $U=[0,1]$ [30].

In order to simplify description of operations, we assume that a membership is determined by two functions:

$$
J_{x}=\left[\underline{\mu}_{\tilde{A}}(x, z), \bar{\mu}_{A}^{\sim}(x, z)\right],
$$

where $\underline{\mu}_{A}^{\sim}(x, z)$ is a lower membership function determining the lower boundary of the membership function, $\bar{\mu}_{A}^{\sim}(x, z)$ is a upper membership function determining the upper boundary of the membership function. 
Similarly to the 3-D fuzzy set and the fuzzy set of Type $2[25,27]$, we determine basic operations for 3-D IT2 FS as operations for IT2 FS considering the available spacious coordinate.

In [25], the 3-D fuzzy set is created to process spacious data. On its basis, a fuzzy controller has been developed to control processes with distributed parameters. Yet, this controller applies a crisp membership function that greatly limits the system and requires accurate expert evaluation, which is impossible for ultrasonic cleaning. That is why, to form adaptive automatic control, a controller based on the 3-D IT2 FS controller that enables combining advantages of the 3-D controller and the one based on IT1 FS is developed.

The developed 3-D IT2 FS controller possesses the same structure as the basic one [32], yet, standard operations of fuzzification, the fuzzy logic output and defuzzification in a more detailed description differ greatly (Fig. 2). Let us consider each stage in detail.

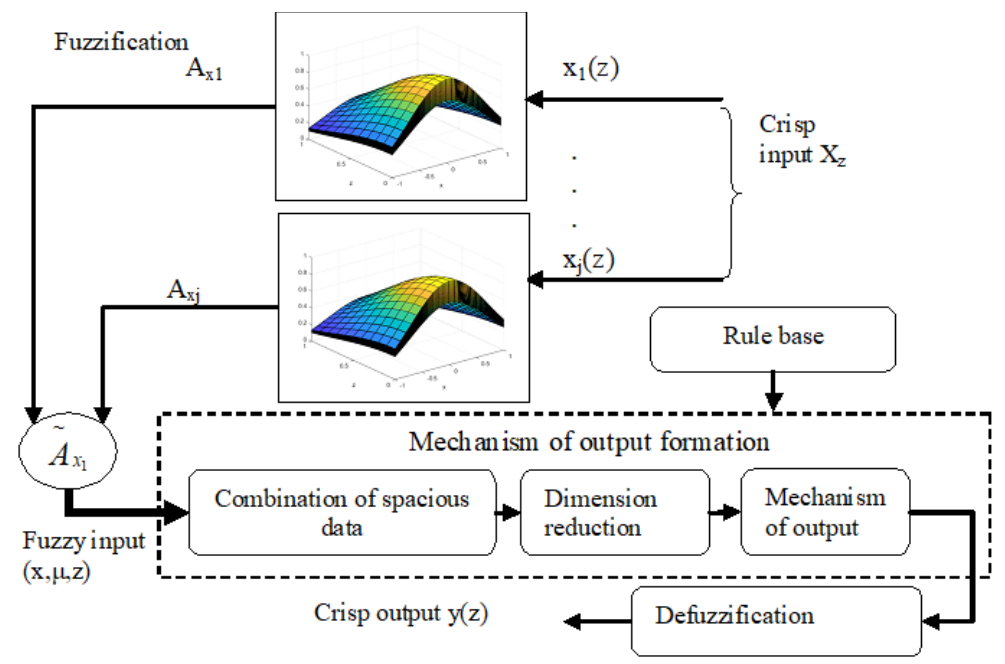

Fig. 2. The 3-D IT2 FS 3-D IT2 FS-based controller.

Fuzzification is the first stage of data processing. Similarly to the traditional fuzzy logic, fuzzification can be performed in various ways and depends on the task to be solved.

To make it simpler and more concise, all the operations of the controller are presented for solo-set fuzzification. The membership function for solo-set fuzzification looks in the following way

$$
\mu_{\tilde{A}}(x, z)=\left\{\begin{array}{l}
{\left[\mu_{\tilde{A}}(x), \bar{\mu}_{A}^{\sim}(x)\right], x=x^{\prime}, z=z^{\prime} .} \\
0, x \neq x^{\prime}, z \neq z^{\prime}
\end{array} .\right.
$$

In this case, fuzzification for each crisp input $X$ is determined in the following way [25]:

$$
\begin{aligned}
& \tilde{A}_{x_{1}}=\sum_{z \in Z} \sum_{x_{1}(z) \in X_{1}} \frac{\mu_{x_{1}}\left(x_{1}(z), z\right)}{\left(x_{1}(z), z\right)} \text {, where } \mu_{x_{1}}\left(x_{1}, z\right)=\int_{u \in J_{x 1}}\left[\underline{\mu}_{A}^{\sim}\left(x_{1}(z), z\right), \bar{\mu}_{A}^{\sim}\left(x_{1}(z), z\right)\right] \frac{1}{u}, \\
& \vdots \\
& \tilde{A}_{x_{N}}=\sum_{z \in Z x_{1 N}(z) \in X_{N}} \frac{\mu_{x_{N}}\left(x_{N}(z), z\right)}{\left(x_{N}(z), z\right)} \text {, where } \mu_{x_{N}}\left(x_{N}, z\right)=\int_{u \in J_{x N}}\left[\underline{\mu}_{A}^{\sim}\left(x_{N}(z), z\right), \bar{\mu}_{A}^{\sim}\left(x_{N}(z), z\right)\right] \frac{1}{u}
\end{aligned}
$$

where $u$ is the secondary variable which is an interval for the membership function with set 
$x$ and $z$.

Thus, general fuzzification for $N$ crisp inputs looks like:

$$
\tilde{A}_{x_{1}=}=\sum_{z \in Z} \sum_{x_{1}(z) \in X_{1}} \ldots \sum_{x_{N}(z) \in X_{N}} \frac{\mu_{1}\left(x_{1}(z), z\right) * \ldots * \mu_{1}\left(x_{N}(z), z\right)}{\left(x_{1}(z), \ldots, x_{N}(z), z\right)},
$$

where the t-norm operation is marked by *.

After fuzzification of crisp inputs, we proceed to forming the fuzzy logic output based on the set rule base. By using 3-D IT2 FS, we write the $l$-th rule into the base as [25]:

$$
\tilde{R}^{l}: \text { If } x_{1}(z) \text { is } \tilde{C}_{1}^{l} \text { and } \ldots \text { and } x_{J}(z) \text { is } \tilde{C}_{J}^{l},(z) \text { is } \tilde{K}^{l},
$$

where $\widetilde{R}^{l}-1$ is the rule $(l=1,2 \ldots \mathrm{N}) ; x_{j}(z)(j=1,2, \ldots, J)$ are input variables in set points of space, $\tilde{C}_{i}^{l}-3$-D IT2 FS, u is a controlling impact, $\tilde{K}^{l}$ is IT2 FS, N is the number of fuzzy rules.

The fuzzy logic output implies reduction of 3-D IT2 FS to the traditional IT2 FS. The following operations are applied - combination of spacious data, reduction of dimension and formation of an output. Output formation includes operations of combination, intersection and addition described above. Rule (10) can be described as a fuzzy ratio [25]:

$$
\tilde{R}^{l}: \tilde{A}_{1}^{l} \times \ldots \times \tilde{A}_{n}^{l} \rightarrow \tilde{K}^{l}, l=1,2, \ldots, N .
$$

Combination of spacious data is the first operation in the fuzzy output that will lead to transformation of the 3-D fuzzy interval input $\tilde{A}_{x_{1}}$ into the 3-D set $W^{l}$, which is an interval fuzzy set of Type 2 in each point $x_{z}$. The set $W^{l}$ is determined as an expanded sup-star [25] composition of input sets. Fig. 3 presents the composition for two crisp inputs for the space $Z: x(z)=\left[x_{1}(z), x_{2}(z)\right]$. The expanded sup-star composition performed on the input set and the assigned sets of the $l$-th rule is denoted by:

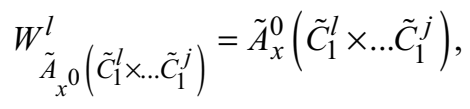

with the upper value of the membership function:

$$
\begin{aligned}
& \bar{\mu}_{W^{l}}=\bar{\mu}_{\tilde{A}_{x^{0}}\left(\tilde{C}_{1}^{\prime} \times \ldots \tilde{C}_{1}^{j}\right.}\left(x_{z}, z\right)=\sup _{x_{1}(z) \in X_{1}, \ldots, x_{j}(z) \in X_{j}}\left[\bar{\mu}_{\tilde{A}_{x}}\left(x_{z}, z\right) * \bar{\mu}_{\tilde{C}_{1}^{\prime} \times \ldots \tilde{C}_{J}^{l}}^{l}\left(x_{z}, z\right)\right]= \\
& \sup _{x_{1}(z) \in X_{1}, \ldots, x_{j}(z) \in X_{j}}\left[\begin{array}{l}
\bar{\mu}_{x_{1}}\left(x_{1}(z), z\right) * \ldots * \bar{\mu}_{x_{j}}\left(x_{j}(z), z\right) * \bar{\mu}_{C_{1}^{\prime}}^{\prime}\left(x_{1}(z), z\right)^{*} \ldots \\
* \bar{\mu}_{C_{J}^{\prime}}^{\prime}\left(x_{J}(z), z\right)
\end{array}\right]= \\
& \left\{\sup _{x_{1}(z) \in X_{1}}\left[\bar{\mu}_{X_{1}}\left(x_{1}(z), z\right) * \bar{\mu}_{C_{1}^{\prime}}^{\prime}\left(x_{1}(z), z\right)\right]\right\} * \ldots \\
& *\left\{\sup _{x_{j}(z) \in X_{j}}\left[\bar{\mu}_{x_{j}}\left(x_{j}(z), z\right) * \bar{\mu}_{\tilde{C}_{j}^{\prime}}^{\prime}\left(x_{j}(z), z\right)\right]\right\},
\end{aligned}
$$

where $z \in Z$, the product $*$ denotes the $t$-norm operation. The lower value of the membership function is determined in a similar way. 


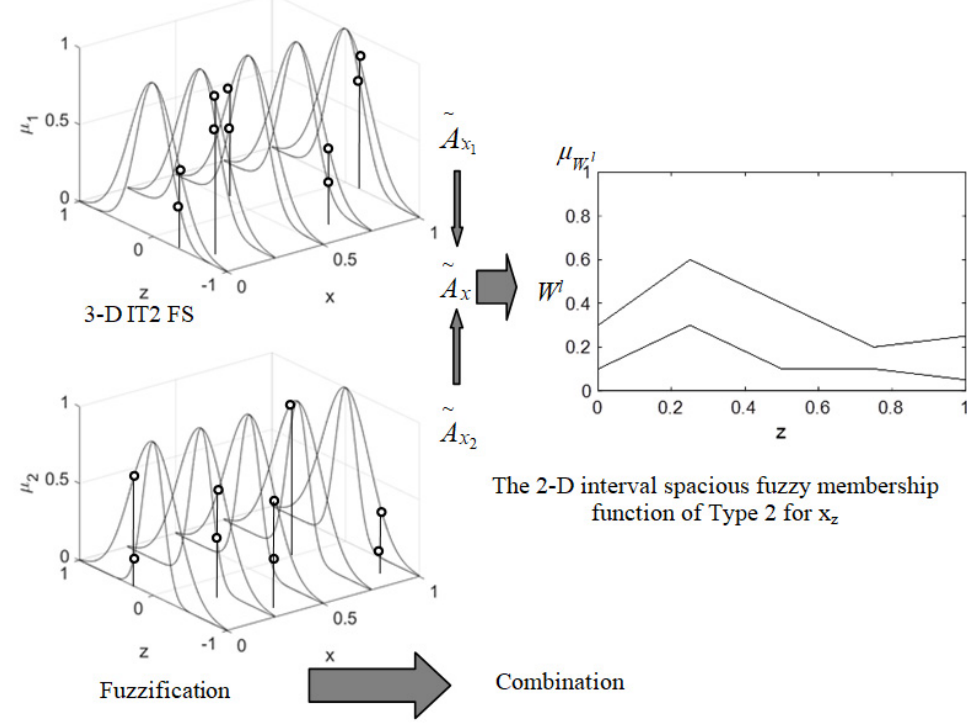

Fig. 3. Combining spacious data for each crisp input $x(z)=\left[x_{1}(\mathrm{z}), x_{2}(z)\right]$.

Next, the operation of dimension reduction is performed. The set $W^{l}$ is spacious distribution for each input $x_{z}$ where the coordinate $z$ is determined on the designing stage. The operation of dimension reduction implies compression of space-distributed data to obtain the two-dimensional one $\left(x_{z}, \mu\right)$.

After combining, we obtain a two-dimensional membership function for each input $x_{z}$ in the plane $(\mu, z)$. The centroid operation is one of the ways to compress the 3-D set $W^{l}$ to the 2-D set that allows describing general impact of the space-distributed system on the input $x_{z}$. The upper and lower membership functions are determined in the following way [33]:

$$
\bar{\mu}_{\varphi^{l}}=\frac{\sum_{i=1}^{p-1} \frac{\left(\bar{\mu}_{W^{l}}\left(z_{i}\right)+\bar{\mu}_{W^{l}}\left(z_{i+1}\right)\right)}{2} \Delta s_{i}}{\sum_{i=1}^{p-1} \Delta s_{i}}, \underline{\mu}_{\varphi^{l}}=\frac{\sum_{i=1}^{p-1} \frac{\left.\underline{\underline{\mu}} W_{W^{l}}\left(z_{i}\right)+\underline{\mu}_{W^{l}}\left(z_{i+1}\right)\right)}{2} \Delta s_{i}}{\sum_{i=1}^{p-1} \Delta s_{i}},
$$

where $p$ is the number of input values of sensors with lower $\underline{\mu}_{W^{l}}\left(z_{i}\right)$ and upper $\bar{\mu}_{W^{l}}\left(z_{i}\right)$ values of the membership function; $\Delta s_{i}=\sqrt{\left(\mu_{W^{l}}\left(z_{i+1}\right)-\mu_{W^{l}}\left(z_{i}\right)\right)^{2}+\left(z_{i+1}-z_{i}\right)^{2}}$ is the length of the $i$-th section of the straight line; $\left(\mu_{W^{l}}\left(z_{i+1}\right)-\mu_{W^{l}}\left(z_{i}\right)\right) / 2$ is the distance from the axis $\mathrm{z}$ to the centroid of the $i$-th segment.

The output mechanism includes accumulation and reduction operations. The result of accumulating $N$ rules looks like [34-35]:

$$
\tilde{B}=\frac{1}{\bigcup_{\forall u \in U}\left[\underline{\mu}_{B}^{\sim}(u), \bar{\mu}_{B}^{\tilde{N}}(u)\right]}=\left(\bigcup_{\forall u \in U}\left[\begin{array}{c}
\left.\max \left(\bar{\mu}_{\varphi^{1}}(u) \ldots \bar{\mu}_{\varphi^{N^{\prime}}}(u)\right),\right] \\
\max \left(\underline{\mu}_{\varphi^{1}}(u) \ldots \underline{\mu}_{\varphi^{N^{\prime}}}(u)\right)
\end{array}\right]\right)^{-1},
$$

where $N^{\prime}$ is the number of production rules. 
Then the upper and lower boundaries of the membership function are determined as

$$
\begin{aligned}
& \underline{\mu}_{B}^{\sim}(u)=\max \left(\underline{\mu}_{\varphi^{1}}(u) \ldots \underline{\mu}_{\varphi^{N^{\prime}}}(u)\right), \forall u \in U ; \\
& \bar{\mu}_{B}^{\sim}(u)=\max \left(\underline{\mu}_{\varphi^{1}}(u) \ldots \underline{\mu}_{\varphi^{N^{\prime}}}(u)\right), \forall u \in U .
\end{aligned}
$$

The type reduction is aimed at proceeding to the traditional fuzzy set. According to [35], we reduce the types by finding the centroid of all the production rules. This results in correlation of numbers containing the maximum and the minimum values:

$$
C_{\tilde{B}}(z)=\frac{1}{\left\{c_{l}(z), \ldots, c_{r}(z)\right\}},
$$

where

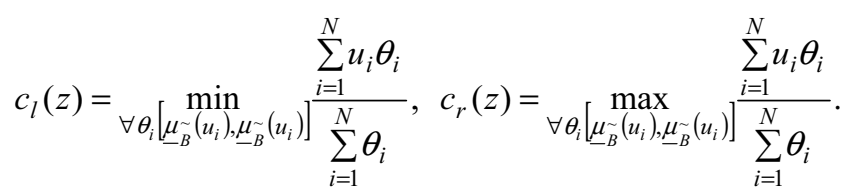

To find $c_{l}$ and $c_{r}$, we apply the iteration Karnik-Mendel algorithm [36] with the following formulae:

$$
c_{l}(z)=\frac{\sum_{i=1}^{L} u_{i}(z) \bar{\mu}_{B}^{\sim}\left(u_{i}\right)+\sum_{i=L+1}^{N} u_{i} \underline{\mu}_{B}^{\sim}\left(u_{i}\right)}{\sum_{i=1}^{L} \bar{\mu}_{B}^{\sim}\left(u_{i}\right)+\sum_{i=L+1}^{N} \underline{\mu}_{\tilde{B}}\left(u_{i}\right)}, \quad c_{r}(z)=\frac{\sum_{i=1}^{R} u_{i} \underline{\mu}_{\tilde{B}}\left(u_{i}\right)+\sum_{i=R+1}^{N} u_{i} \bar{\mu}_{B}^{\sim}\left(u_{i}\right)}{\sum_{i=1}^{R} \underline{\mu}_{\tilde{B}}\left(u_{i}\right)+\sum_{i=R+1}^{N} \bar{\mu}_{B}^{\sim}\left(u_{i}\right)} .
$$

Defuzzification is the final stage. As reduction of types is accompanied by centroid determination, defuzzification implies [35]:

$$
u=\frac{1}{2}\left(c_{r}+c_{l}\right) .
$$

\section{Results and discussion}

The values of the final change of ultrasonic responses recorded in set time intervals $t$ and those of the current ultrasonic response are used as a crisp input. Times intervals are selected so that to reduce the impact of deviations produced by multiple dispersion of ultrasonic waves while the ultrasonic cleaning radiators are in service.

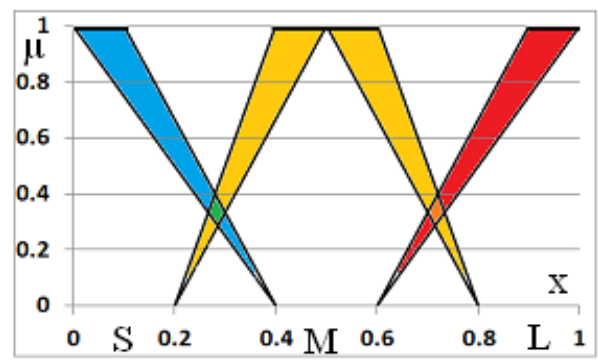

Fig. 4. The membership function for $\Delta x_{i}, x_{i}$ and $u_{i}$; and the rule base. 
Let four sensors be set with positions $z=\left(\begin{array}{llll}0 & 0.33 & 67 & 1\end{array}\right)$ and eight ultrasonic radiators $z=\left(\begin{array}{lllllll}0 & 0.17 & 0.33 & 0.5 & 0.67 & 0.83 & 1\end{array}\right)$. Then, the input data $\Delta x_{z}=\left(\Delta x_{z 1}, \Delta x_{z 2}, \ldots, \Delta x_{z 4}\right)$ and $x_{z}=\left(x_{z 1}, x_{z 2}, \ldots x_{z 4}\right)$ are normalized spacious inputs to denote the difference between the two final measurements $\left(\Delta x_{\mathrm{z}}=\left|x_{z}-x_{z}^{(1)}\right|\right)$ and the value of the final measurement respectively. The input data are $u_{z}=\left(u_{z 1}, u_{z 2}, \ldots, u_{z 8}\right)$. In the general case, the ratio of the number of sensors and ultrasonic radiators is determined by the size of the ultrasonic tank and capacity of selected radiators. All the input variables $\Delta x_{z}$ and $x_{z}$ and output variables $\mathrm{u}$ are normalized on $[0,1]$. All the $\Delta x_{z}$ and $x_{z}$, as well as the output $u_{z}$ are classified into three linguistic marks: large $(L)$, medium $(M)$ and small $(S)$. For the sake of convenience, the membership function is selected as an interval trapezium as is shown in Fig. 4. The rule base (Table 1) is formed as follows: we suppose that the maximum impact results from changes in sensor readings, while the signal itself can be conditioned not only by the product's contamination rate, but also by its geometry and position in the tank.

Table 1. Rule base.

\begin{tabular}{|l|l|l|l|}
\hline$\Delta x_{i} / x_{i}$ & $\tilde{S}$ & $\tilde{M}$ & $\tilde{L}$ \\
\hline$\tilde{S}$ & $\tilde{M}$ & $\tilde{S}$ & $\tilde{S}$ \\
\hline$\tilde{M}$ & $\tilde{M}$ & $\tilde{M}$ & $\tilde{S}$ \\
\hline$\tilde{L}$ & $\tilde{L}$ & $\tilde{L}$ & $\tilde{M}$ \\
\hline
\end{tabular}

We calculate a result for each $u_{z}=\left(u_{z 1}, u_{z 2}, \ldots u_{z 8}\right)$ separately. As it is necessary to consider several controlling outputs with spacious dependency on inputs, formulae (14) for the current $u_{z 0}$ with the spacious coordinate $\mathrm{z}=\mathrm{Z}_{0}$ are reduced to the following form ( for $\bar{\mu}_{\varphi^{l}}$, while for $\underline{\mu}_{\varphi^{l}}$ the changes are similar):

$$
\bar{\mu}_{\phi^{l}}=\frac{\sum_{i=1}^{p-1} \frac{\left(\bar{\mu}_{W^{l}}\left(z_{i}\right)|2| z_{i}-z_{0}|-1|+\bar{\mu}_{W^{l}}\left(z_{i+1}\right)|2| z_{i+1}-z_{0}|-1|\right)}{2} \Delta s_{i}}{\sum_{i=1}^{p-1} \Delta s_{i}} .
$$

All the other actions are performed according to the algorithm of the controller described above. They result in the intensity obtained for each radiator enabling the product's insonation.

While modelling ultrasonic cleaning with the control based on the developed controller, power consumption has been reduced by $12.46 \%$ as compared with the control with time only limitations. The period of ultrasonic cleaning has been reduced by $8.23 \%$.

\section{Conclusions}

There are developed new fuzzy methods of control intended for spacious distributed systems with input data difficult to be assessed through expert evaluation. The controller is based on combining the 3-D fuzzy set and the interval fuzzy set of Type 2. The developed controller differs from the traditional one by reduction of 3-D and that IT2 FS Type to IT1 FS Type. The rule base remains two-dimensional and is not dependent on the number of sensors. On the basis of the described methods, the model of the 3-D IT2 FS controller is developed and applied to cleaning mining machines. Spacious distribution of the process and ambiguity of expert evaluation are considered while determining the course of the process according to the data of ultrasonic sensors. 
The authors express their sincere gratitude to Kryvyi Rih National University for support in conducting research.

\section{References}

1. Yamashita, T., \& Ando, K. (2018). Low-intensity ultrasound induced cavitation and streaming in oxygen-supersaturated water: Role of cavitation bubbles as physical cleaning agents. Ultrasonics Sonochemistry, (52), 268-279. https://doi.org/52. 10.1016/j.ultsonch.2018.11.025

2. Niemczewski, B. (2007). Cavitation intensity of water under practical ultrasonic cleaning conditions. Ultrasonics sonochemistry, (14), 13-8. https://doi.org/10.1016/j.ultsonch.2005.11.009

3. Saalbach, K.-A.,Twiefel, J., \& Wallaschek, J. (2018). Self-Sensing Cavitation Detection in Ultrasound-Induced Acoustic Cavitation. Ultrasonics, (94), 401-440. https://doi.org/10.1016/j.ultras.2018.06.016

4. Xu, H., Tu, J., Niu, F. \& Yang, P. (2016). Cavitation dose in an ultrasonic cleaner and its dependence on experimental parameters. Applied Acoustics, (101), 179-184. https://doi.org/10.1016/j.apacoust.2015.08.020

5. Duran, F., \& Teke, M. (2018). Design and implementation of an intelligent ultrasonic cleaning device. Intelligent Automation and Soft Computing, 1-10. https://doi.org/10.31209/2018.11006161

6. Rahim A, Bargoshadi H., \& Sarrafi S. (2011). Design and Manufacture an Ultrasonic Dispersion $\begin{array}{lllll}\text { System. Sensors } \& \text { Transducers Journal, } & \text { 126(3), }\end{array}$ https://doi.org/10.1109/CSPA.2011.5759903

7. Roohi, R., Abedi, E., Hashemi, S. M. B., Marszałek, K., \& Barba, F. (2019). Ultrasound-assisted bleaching: Mathematical and 3D computational fluid dynamics simulation of ultrasound parameters on microbubble formation and cavitation structures. Innovative Food Science \& Emerging Technologies, (55), 66-79. https://doi.org/55. 10.1016/j.ifset.2019.05.014

8. Tangsopha, W., Thongsri, J. \& Busayaporn, W. (2017). Simulation of ultrasonic cleaning and ways to improve the efficiency. 2017 International Electrical Engineering Congress. https://doi.org/10.1109/IEECON.2017.8075747

9. Chahine, G. L., Kapahi, A., Choi, J.-K., \& Hsiao, C.-T. (2016). Modeling of Surface Cleaning by Cavitation Bubble Dynamics and Collapse. Ultrasonic Sonochem, (29), 528-549. https://doi.org/10.1016/j.ultsonch.2015.04.026

10. Treeby, B., \& Cox, B.T. (2010). k-Wave: MATLAB toolbox for the simulation and reconstruction of photoacoustic wave fields. Journal of Biomedical Optics, 15(2), 021314-1021314-12. https://doi.org/15. 021314. 10.1117/1.3360308

11. Morkun, V., Morkun, N., \& Pikilnyak, A. (2014). Modeling of ultrasonic waves propagation in inhomogeneous medium using fibered spaces method (k-space). Metallurgical and Mining Industry, (6), 43-48.

12. Treeby, B.E., Jaros, J., Rendell, A.P., \& Cox, B.T. (2012). Modeling nonlinear ultrasound propagation in heterogeneous media with power law absorption using a k-space pseudospectral method. The Journal of the Acoustical Society of America, 131(6), 4324-4336. https://doi.org/10.1121/1.4712021

13. Ray, W.H. (1981). Advanced process control. New York: McGraw-Hill.

14. Morkun, V., \& Morkun, N. (2018). Estimation of the crushed ore particles density in the pulp flow based on the dynamic effects of high-energy ultrasound. Archives of Acoustics, (43), 61-67. https://doi.org/10.24425/118080

15. Morkun, V., Tron, V., \& Paraniuk, D. (2015). Formation of rock geological structure model for drilling process adaptive control system. Metallurgical and Mining Industry, (7), 12-15.

16. Morkun, V., Morkun, N., \& Tron. V. (2015). Identification of control systems for ore-processing industry aggregates based on nonparametric kernel estimators. Metallurgical and Mining Industry, (1), 14-17. 
17. Morkun, V., Morkun, N., \& Tron. V. (2015). Distributed closed-loop control formation for technological line of iron ore raw materials beneficiation. Metallurgical and Mining Industry, (7), 18-19.

18. Morkun, V., Morkun, N., \& Tron. V. (2015). Distributed control of ore beneficiation interrelated processes under parametric uncertainty. Metallurgical and Mining Industry, (8), 18-21.

19. Morkun, V., Morkun, N., \& Tron, V. (2015). Model synthesis of nonlinear nonstationary dynamical systems in concentrating production using Volterra kernel transformation. Metallurgical and Mining Industry, (7), 6-9.

20. Morkun, V., \& Tron, V. (2014). Automation of iron ore raw materials beneficiation with the operational recognition of its varieties in process streams. Metallurgical and Mining Industry, (6), 4-7.

21. Morkun, V., Morkun, N., \& Pikilnyak, A. (2014). The adaptive control for intensity of ultrasonic influence on iron ore pulp. Metallurgical and Mining Industry, (6), 8-11.

22. Tron, V., Tsokurenko, O., Paraniuk, D., \& Haponenko, I. (2019) Formation of the adaptive fuzzy model of the rock geological structure for exploratory drilling. E3S Web of Conferences, (123), 01037. https://doi.org/10.1051/e3sconf/201912301037

23. Mendel, J.M. (1995). Fuzzy logic systems for engineering: a tutorial. Proceedings of the IEEE, 83(3), 345-377. https://doi.org/10.1109/5.364485

24. Rajani C., Klami A., Salmi A., Rauhala T., Hæggström E., \& Myllymäki P. (2018). Detecting industrial fouling by monotonicity during ultrasonic cleaning. 2018 IEEE 28th International Workshop on Machine Learning for Signal Processing (MLSP). https://doi.org/10.1109/MLSP.2018.8517080

25. Li, H.-X., Zhang, X.-X., \& Li, S.-Y. (2007). A Three-Dimensional Fuzzy Control Methodology for a Class of Distributed Parameter Systems. IEEE Transactions on Fuzzy Systems, 15(3), 470481. https://doi.org/10.1109/TFUZZ.2006.889962

26. Zhang, X.-X., Fu, Z.-Q., Li, S.-Y., Zou, T., \& Wang, B. (2017). A time/space separation based 3D fuzzy modeling approach for nonlinear spatially distributed systems. International Journal of Automation and Computing, 15(1), 52-65. https://doi.org/10.1007/s11633-017-1080-0

27. Zadeh, L.A. (1975). The concept of a linguistic variable and its application to approximate reasoning-I. Information Sciences, 8(3), 199-249. https://doi.org/10.1016/0020-0255(75)90036-5

28. Lee, C. C. (1990). Fuzzy logic in control systems: fuzzy logic controller. I. IEEE Transactions on Systems, Man, and Cybernetics, 20(2), 404-418. https://doi.org/10.1109/21.52551

29. Zhang, X., Li, S., \& Li, H.-X. (2007). Interval-Valued Fuzzy Logic Control for a Class of Distributed Parameter Systems. International Journal of Uncertainty, Fuzziness and KnowledgeBased Systems, (15), 457-481. https://doi.org/10.1142/S0218488507004790

30. Olyzarenko, S.A., Brezhnev, E.V., \& Perepelytsa, A.V. (2010), Nechetkye mnozhestva typa 2. Termynolohyia y predstavlenye. Information Processing Systems, (89), 131-140.

31. Olyzarenko, S.A., Kapranov, A.V. \& Perepelytsa, A.V. (2011). The interval type 2 fuzzy sets. Terminology, representations, operations. Information Processing Systems, 2(92), 39-45.

32. Mamdani, E.H. (1974). Application of fuzzy algorithms for control of simple dynamic plant. Proceedings of the Institution of Electrical Engineers, (121), 1585-1588. https://doi.org/10.1049/piee.1974.0328

33. Liang, Q., \& Mendel, J.M. (2000). Interval type-2 fuzzy logic systems: theory and design. IEEE Transactions on Fuzzy Systems, 8(5), 535-550. https://doi.org/10.1109/91.873577

34. Mendel, J.M., John, R.I., \& Liu, F. (2006). Interval Type-2 Fuzzy Logic Systems Made Simple. IEEE Transactions on Fuzzy Systems, https://doi.org/10.1109/TFUZZ.2006.879986

35. Robert, J. (2002). Embedded interval valued type-2 fuzzy sets. IEEE International Conference on Fuzzy Systems, (2), 1316-1320. https://doi.org/10.1109/FUZZ.2002.1006694

36. Karnik, N.N., \& Mendel, J.M. (2001). Centroid of a type-2 fuzzy set. Journal of Information Science, (132), 195-220. https://doi.org/10.1016/S0020-0255\%2801\%2900069-X 